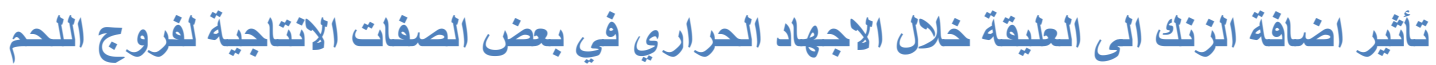

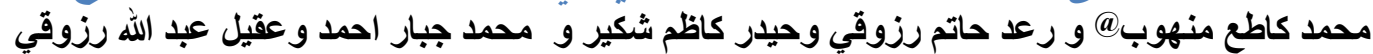

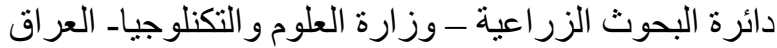

E-Mail: mohammadkatih@yahoo.com

2013 /1/8: قبل للنشر

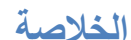

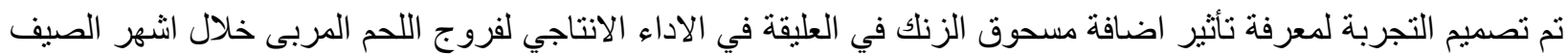

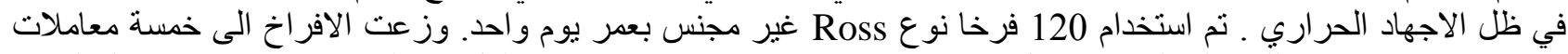

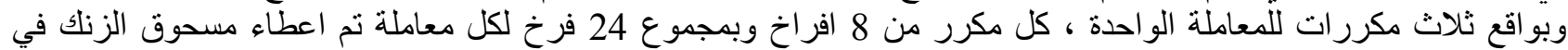

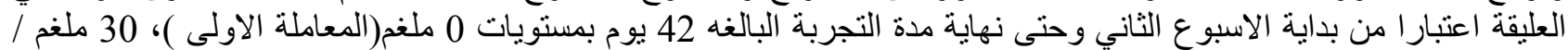

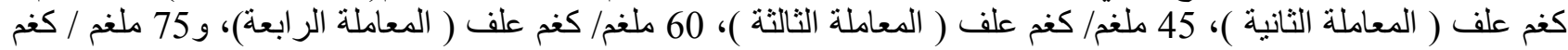

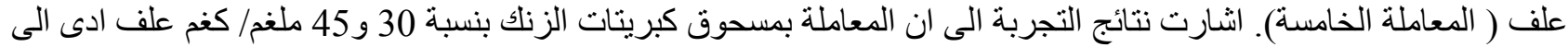

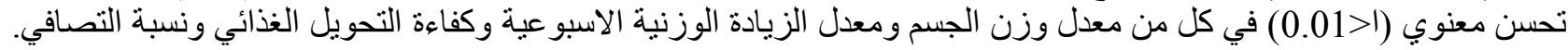

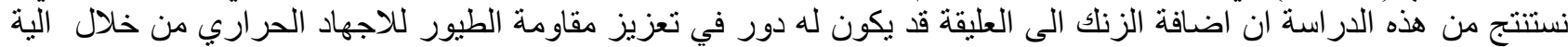
عمله كمانع للاكسدة.

الكلمات المفتاحية :- فروج اللحم, زنتك,الاجهاد الحراري.

مستويات مختلفة من الزنك الى العليقة في الاداء الانتاجي لفروج اللحم في ظروف الاجهاد الحر اري.

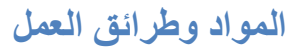

أجريت هذه الدراسة في حقل الطيور الداجنه التابع لقسم

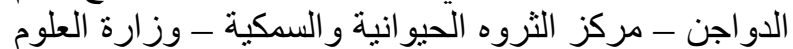

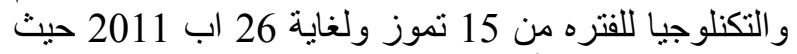

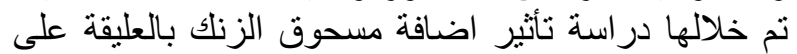

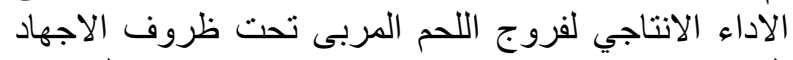

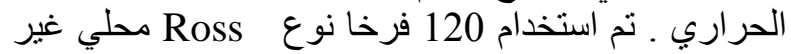
مجنس بعمر يوم واحد وقد جهزت الإن الافر اخخ من احد مفاقس

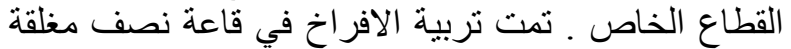
بطريقة التربية الارضية اذ قسمت القاعة القاعة الى 15 حجره

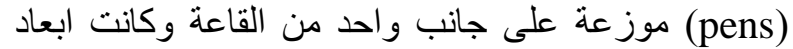

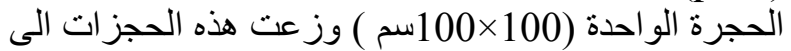

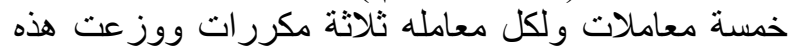

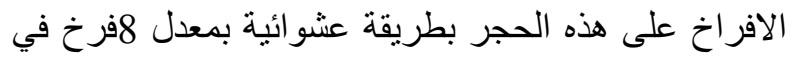

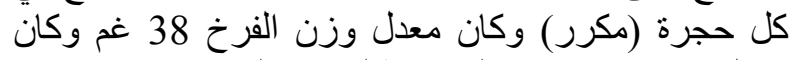

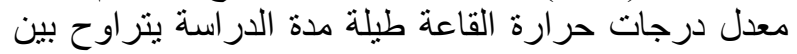

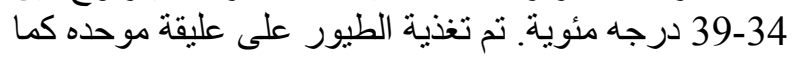

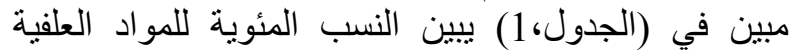

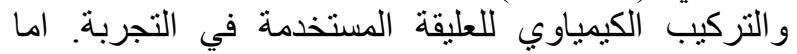
المعاملات الغذائية المستخدمة في التجربة والتئية التي استخدم فيها مسحوق الزنك فقد بدأت من عمر 7 ايام كما يلي:-

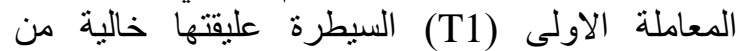
مسحوق الزنك.المعاملة الثانية (T2) اضيف الكيف الى الى عليقتها مسحوق الزنك بنسبة 30 ملغ /كغ علفة الفية المعاملة الثالثة

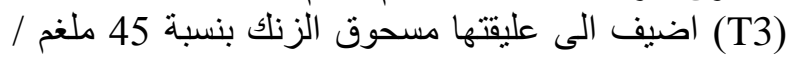

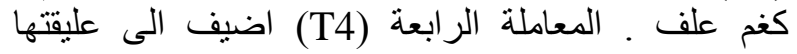

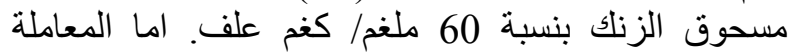
الخامسة (T5) اضيف الى عليقتها مسحوق الزنك لك بنسبة 75 ملغم / كغم علف.

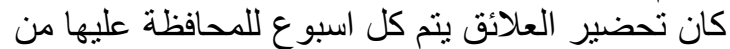
التلف وكان يتم اضافة الكمية المقررة من الزنانك التى كل للى

\section{المقامة المة}

مناطق واسعة من العالم ومنها العراق تتعرض الى الى الى القئ

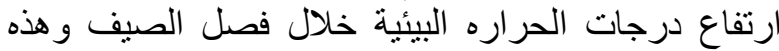
تعتبر مشكله كبيرة وتؤثر سلبيا على الكئية الكفاءه الانتاجية

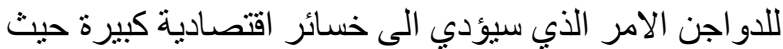

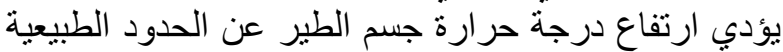

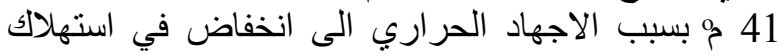

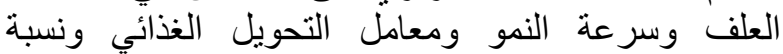
الهلاكات وصفات الذبيحه و انتاج ووزن البيض وليض والئل الصفات

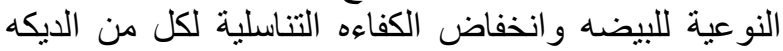

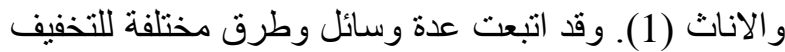

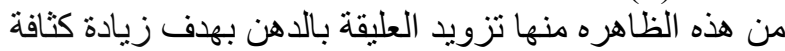

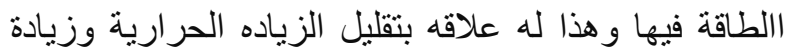
استهلاك الطاقة (2). واعتبر تقليل نسبة البروتينات مع الإنية

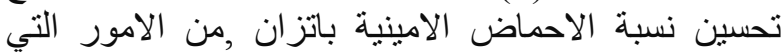

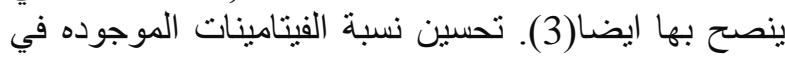
العليقة (4) و المعادن النادره (5) تساعد على تحسين الحالة الكينة

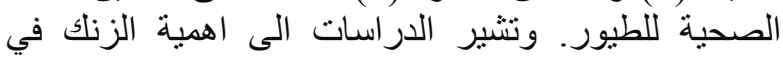

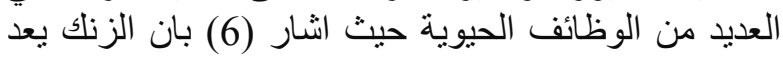

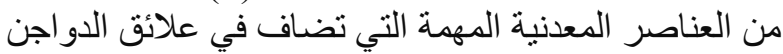

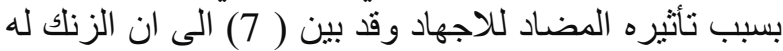

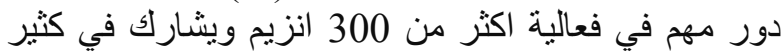
من الفعاليات الانزيمية و الايضية في الجسم. اذئية يؤثر ايجابيا

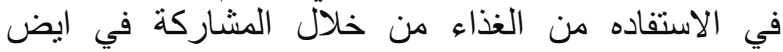
الكاربوهيدرات و الدهون و البروتينات (8) الامر الذي الذي يحسن من الاداء الانتاجي للطيور المرباة في درجات البرات حر اره عالية.

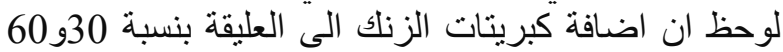

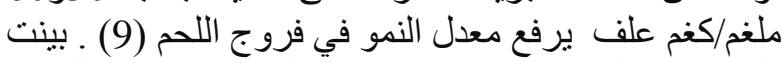

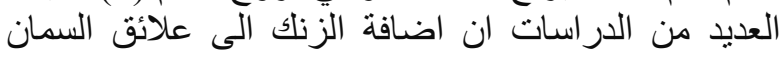

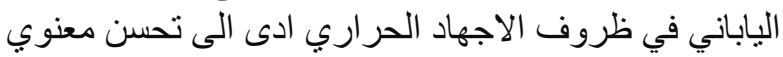

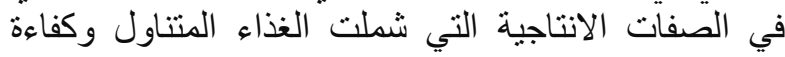

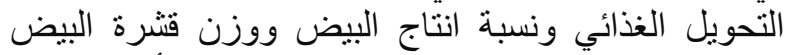

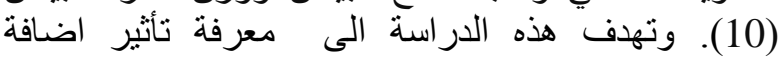


ولمرنبة عشرية واحدة. كذللك تم حساب (Sartorius)

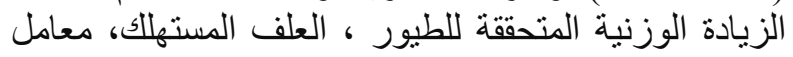

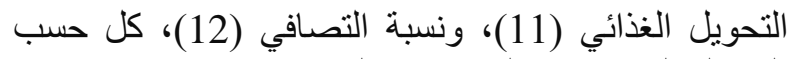

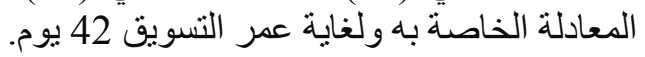

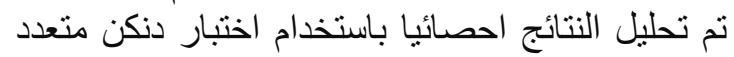

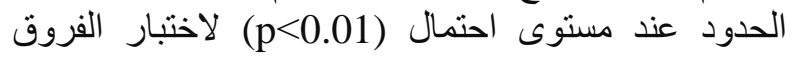
المعنوية بين المنوسطات (13).
عليقة بعد وزنها بميز ان حساس ،اذ كان يتم الخلط اولا بكمية

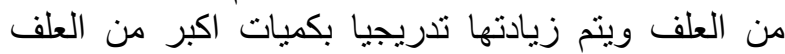

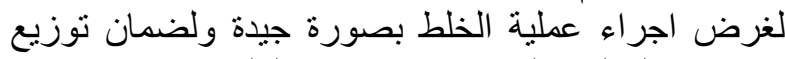

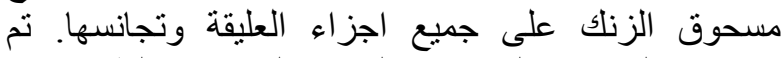

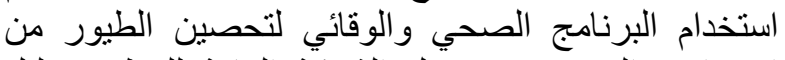

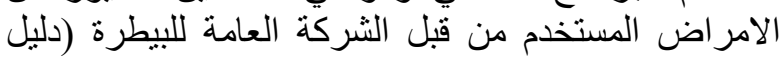

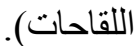
تم دراسة الصفات الانتاجية بحساب الوزن الحي للطيور

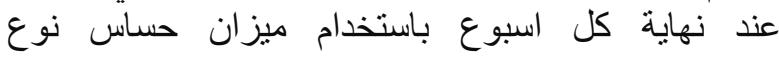

\begin{tabular}{|c|c|}
\hline النسبة المئوية & الماده العلفية \\
\hline 40 & ذره صفراء مستورده \\
\hline 27 & حنطه محلية \\
\hline 22 & كسبة فول الصويا (44\% بروتين خام ) \\
\hline 10 & مركز بروتيني * \\
\hline 0.7 & حجر كلس \\
\hline 0.3 & 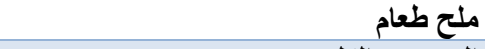 \\
\hline 100 & المجموع الكلي \\
\hline & التركيب الغذائي المحسوب** \\
\hline 2891 & طاقه ممثله ( كيلو سعره / كفم علف ) \\
\hline 21.80 & بروتين خام \\
\hline 132.61 & نسبة الطاقة الى البروتين (C Ratio/P) \\
\hline 0.86 & 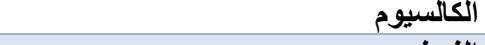 \\
\hline 0.44 & الفسفور \\
\hline 5.12 & الياف خام \\
\hline
\end{tabular}

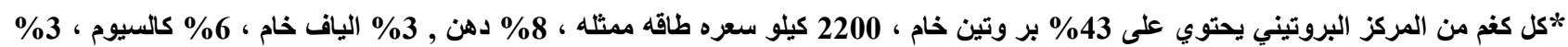

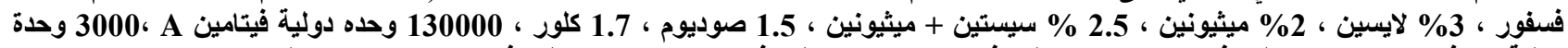

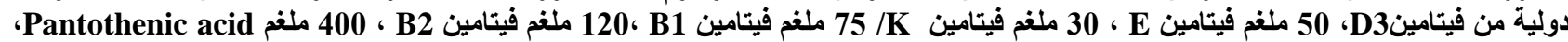

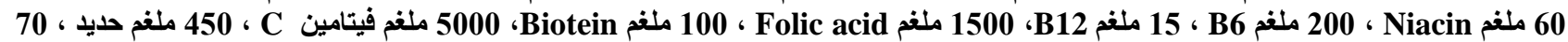

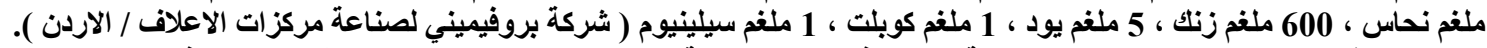

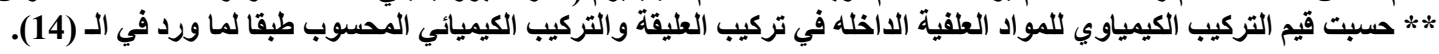

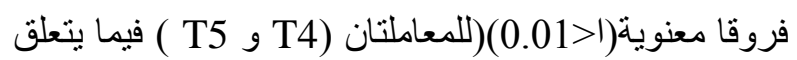

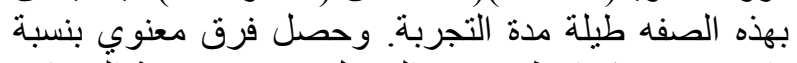

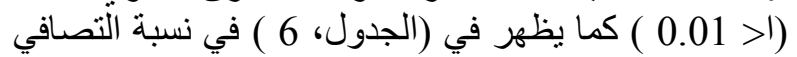

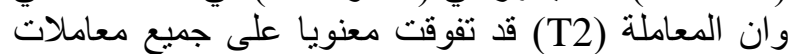

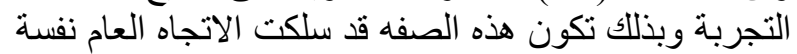
الذي سلكته جميع الصفات الانتاجية الايجابية السابقة الذكر.

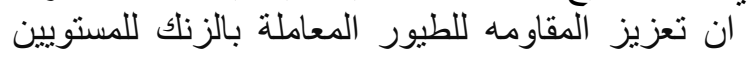

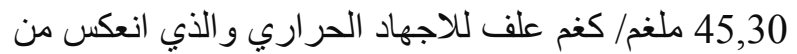

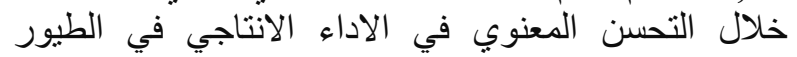

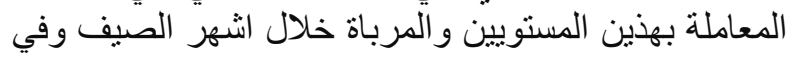

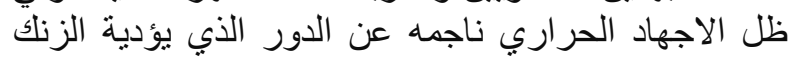

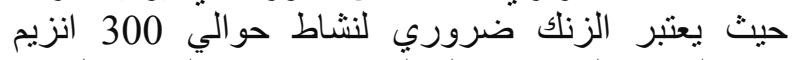

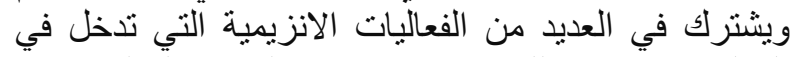

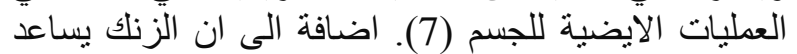

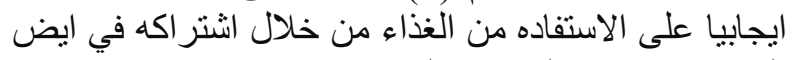

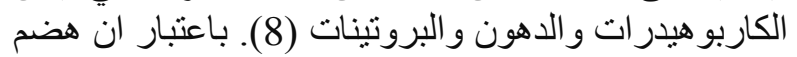

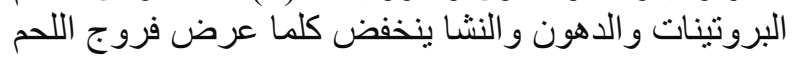

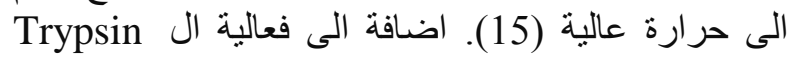
Chymotrypsin

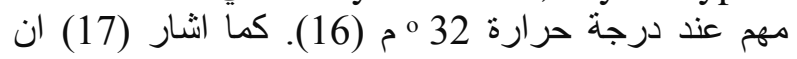

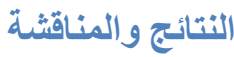

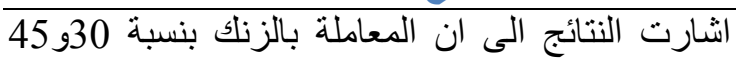

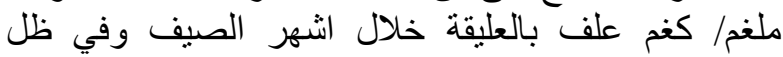

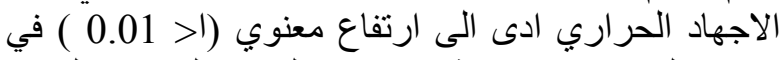

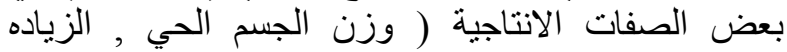

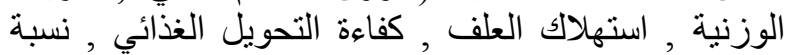

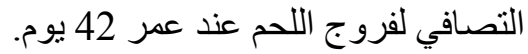

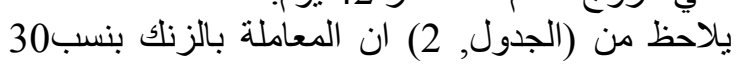

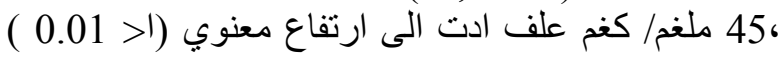

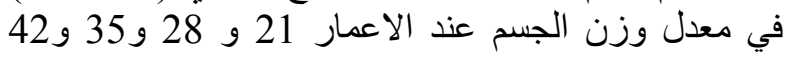

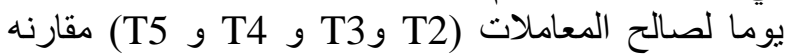

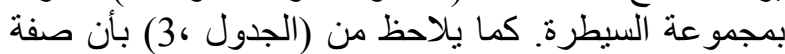

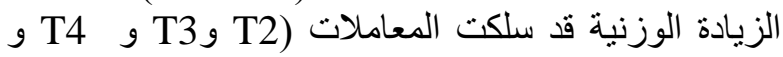

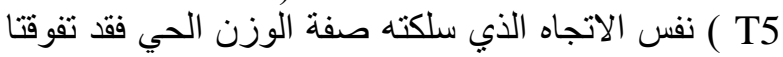

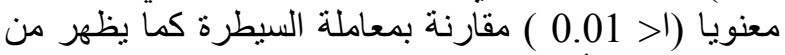

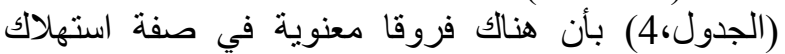

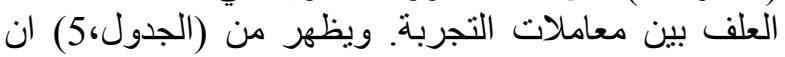

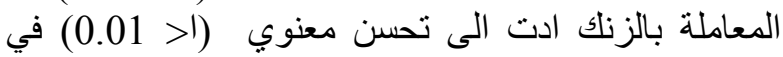

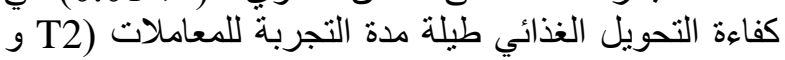

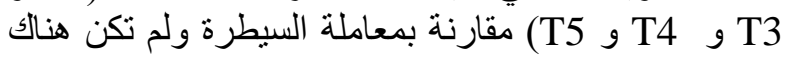


الالبومين مع البلازما الى الكبد ( 21 الكا 22). كذللك هنالك

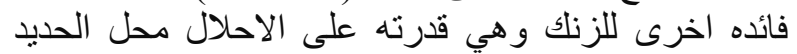

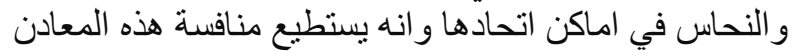

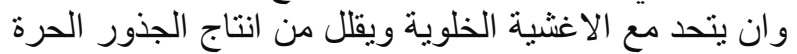

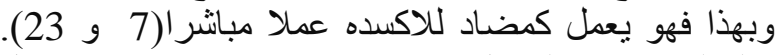
و الزنك يمنع عملية ال(lipid peroxidation) من خلال

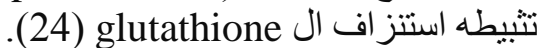
وبذللك تتضح لنا اهية هذا المعدن وبالكميات المناسبة

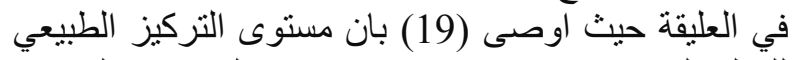

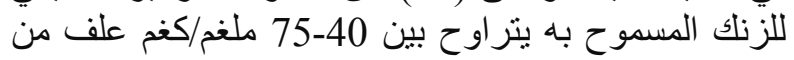

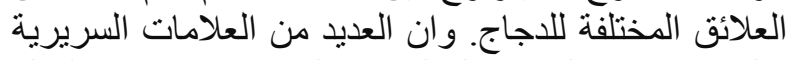

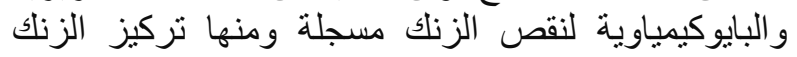

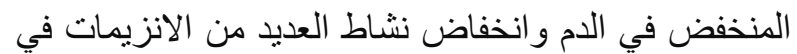

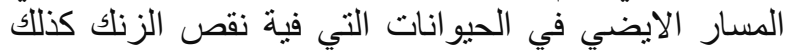

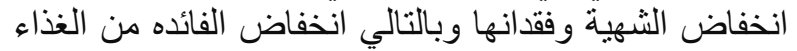

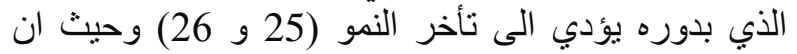

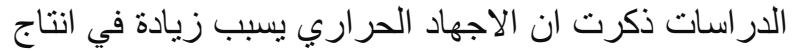
الجذور الحرة (27) وانها تقلل من كمية الفيتامينات

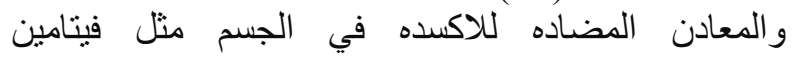

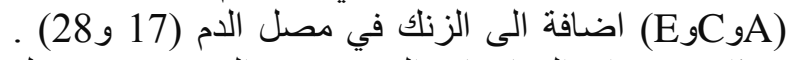

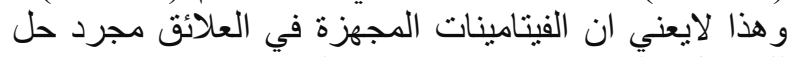

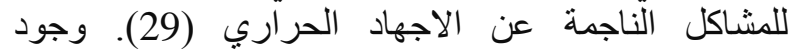

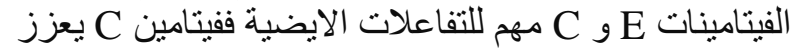

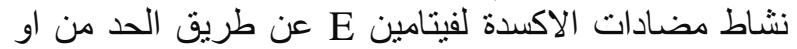
خفض الجذور الحرة (Tocopheroxyl) في شكل تُكل النشاط

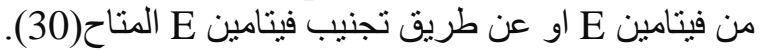

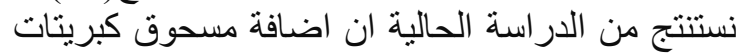

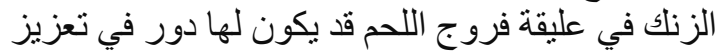

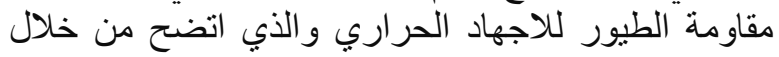

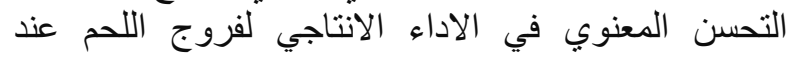
المستويات 30 و 45 ملغم/كغم علف.
الزيادة الخطية في العلف المستهلك وتحسين كفاءة التحويل

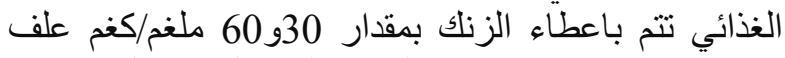

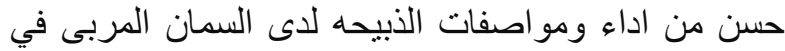

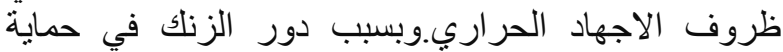
النسيج البنكرياسي من التدمير بفعل العو امل المؤكسده فانه

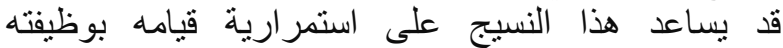

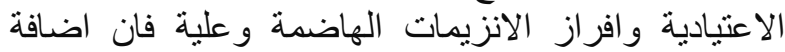

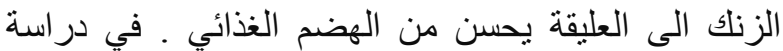

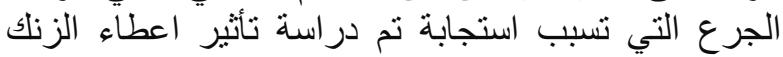

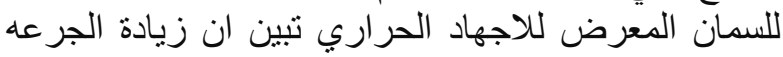

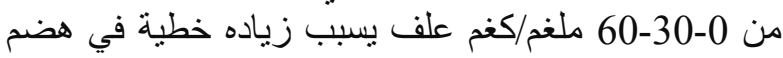
المو اد الغذائية للعليقه و المو اد العضوية فئها و البروتين الخام

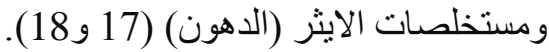

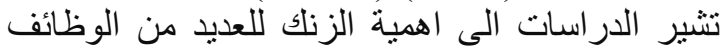

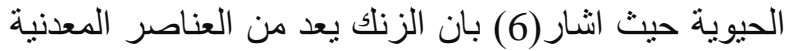

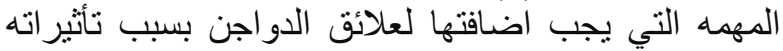

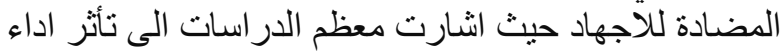

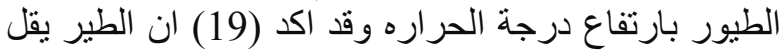

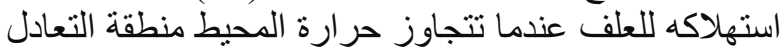

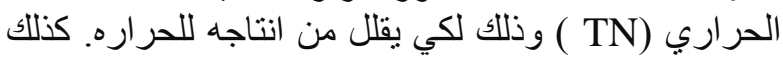

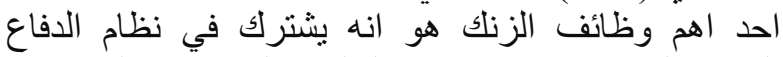

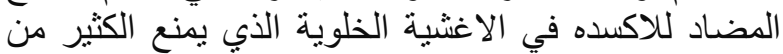

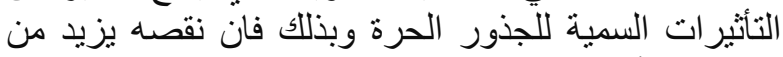
الضرر التأكسدي لاغثية الخلايا التي تسبيها الجذور الحرة ( )

الالية الني يعمل فيها الزنك كمضاد للاكسده غير معروفة

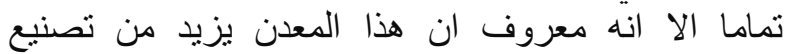

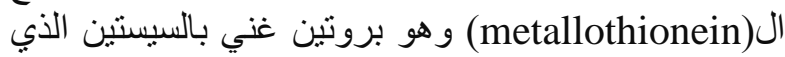

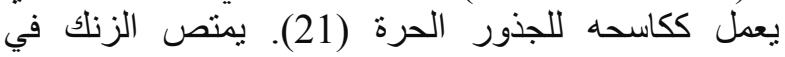

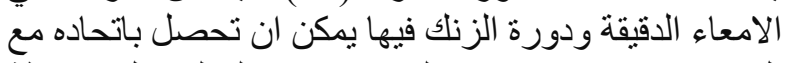

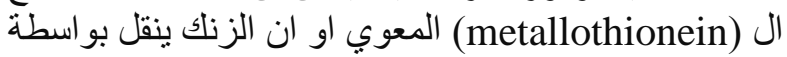

جلول, 2 : تأثير اضافة نسب مختلفة من مسحوق كبريتات الزنك الى العليقة على معدل وزن الجسم ( غم ) لفروج اللحم عند الاعمار المختلفة

\begin{tabular}{|c|c|c|c|c|c|c|}
\hline 42 & 35 & 28 & 21 & 14 & 7 & العمر / باليوم \\
\hline $\begin{array}{c}\text { B } \\
2.07 \pm 1380.00\end{array}$ & $\begin{array}{c}C \\
1.61 \pm 943.30\end{array}$ & $\begin{array}{c}B \\
3.49 \pm 608.33\end{array}$ & $\begin{array}{c}\text { B } \\
1.21 \pm 370.00\end{array}$ & $\begin{array}{c}A \\
1.09 \pm 203.30\end{array}$ & $\begin{array}{c}\text { A } \\
0.44 \pm 92.66\end{array}$ & (control)T1 \\
\hline $\begin{array}{c}\mathrm{A} \\
1.47 \pm 1486.66\end{array}$ & $\begin{array}{c}A \\
0.86 \pm 1003.30\end{array}$ & $\begin{array}{c}A \\
1.15 \pm 644.30\end{array}$ & $\begin{array}{c}\mathrm{A} \\
1.38 \pm 386.60\end{array}$ & $\begin{array}{c}A \\
2.19 \pm 208.00\end{array}$ & $\begin{array}{c}\mathrm{A} \\
1.50 \pm 93.30\end{array}$ & (kg \\
\hline $\begin{array}{c}\mathrm{A} \\
1.50 \pm 1411.40\end{array}$ & $\begin{array}{c}\text { B } \\
0.69 \pm 986.60\end{array}$ & $\begin{array}{c}A \\
1.27 \pm 640.00\end{array}$ & $\begin{array}{c}\mathrm{A} \\
2.13 \pm 386.00\end{array}$ & $\begin{array}{c}A \\
2.02 \pm 206.00\end{array}$ & $\begin{array}{c}\mathrm{A} \\
\mathbf{0 . 5 7} \pm 93.00\end{array}$ & $(\mathrm{~kg} / \mathrm{mg} \mathrm{45)T3}$ \\
\hline $\begin{array}{l}\text { AB } \\
1.95 \pm 1393.30\end{array}$ & $\begin{array}{c}D \\
1.96 \pm 893.30\end{array}$ & $\begin{array}{c}C \\
1.61 \pm 566.60\end{array}$ & $\begin{array}{c}\mathrm{C} \\
1.90 \pm 350.00\end{array}$ & $\begin{array}{c}\text { B } \\
0.92 \pm 194.26\end{array}$ & $\begin{array}{c}A \\
0.65 \pm 93.00\end{array}$ & $(\mathrm{~kg} / \mathrm{mg60}) \mathrm{T} 4$ \\
\hline $\begin{array}{c}C \\
0.75 \pm 1206.70\end{array}$ & $\begin{array}{c}E \\
1.55 \pm 810.00\end{array}$ & $\begin{array}{c}D \\
2.25 \pm 553.30\end{array}$ & $\begin{array}{c}\text { D } \\
0.63 \pm 343.30\end{array}$ & $\begin{array}{c}\text { B } \\
1.73 \pm 196.60\end{array}$ & $\begin{array}{c}\mathrm{A} \\
1.15 \pm 90.00\end{array}$ & (kg/mg75)T5 \\
\hline
\end{tabular}

الحروف المختلفة عموديا تثير الى وجود فروق معنوية بين متوسطات المعاملات عند مستوى احتمال (p) (p.01) 
جدول, 3: تأثير اضافة نسب مختلفة من مسحوق كبريتات الزنك الى العليقة في معدل الزيادة الوزنية (غم) لفروج اللحم عذد الاعمار المختلفة

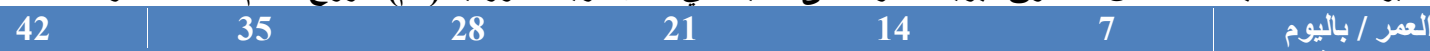

\begin{tabular}{|l|l|l|l|l|l|l|} 
C & AB & C & B & B & AB & (control)T1 \\
\hline
\end{tabular}

\begin{tabular}{|l|l|l|l|l|l|}
$2.39 \pm 1287.33$ & $0.46 \pm 436.70$ & $5.08 \pm 334.96$ & $2.32 \pm 238.3$ & $2.00 \pm 166.70$ & $1.53 \pm 110.63$
\end{tabular}

\begin{tabular}{|l|c|c|c|c|c|c|}
\hline $\mathbf{A}$ & $\mathbf{A}$ & $\mathbf{A}$ & $\mathbf{A}$ & $\mathbf{A}$ & $\mathbf{A}$ & $\mathbf{( k g} / \mathbf{3 0} \mathbf{~ m g}) \mathbf{T} 2$ \\
\hline
\end{tabular}

\begin{tabular}{|c|c|c|c|c|c|}
\hline $1.50 \pm 1393.36$ & $2.05 \pm 483.36$ & $1.04 \pm 359.00$ & $2.54 \pm 257.70$ & $3.12 \pm 178.60$ & $1.94 \pm 114.70$ \\
\hline B & B & B & A & A & A
\end{tabular}
B
$\begin{array}{lll}\mathrm{A} & (\mathrm{kg} / \mathrm{mg} \mathbf{4 5}) \mathrm{T3}\end{array}$

\begin{tabular}{|c|c|c|}
\hline $\mathbf{3 5 9 . 0 0}$ & $\mathbf{2 . 5 4} \pm \mathbf{2 5 7 . 7 0}$ & $\mathbf{3 . 1 2} \pm \mathbf{1 7 8 . 6}$ \\
\hline B & $\mathrm{A}$ & $\mathrm{A}$
\end{tabular}

\begin{tabular}{|l|l|l|l|l|l|}
$2.08 \pm 1318.40$ & $1.97 \pm 424.80$ & $0.57 \pm 346.60$ & $2.98 \pm 254.00$ & $3.60 \pm 180.00$ & $1.80 \pm 113.00$
\end{tabular}

\begin{tabular}{|c|c|c|c|c|c|c|c|} 
B & A & C & C & C & BC & $(\mathrm{kg} / \mathbf{m g 6 0}) \mathbf{T} 4$ \\
\hline
\end{tabular}

\begin{tabular}{|c|c|c|c|c|c|}
\hline $1.95 \pm 1300.70$ & $9.92 \pm 500.00$ & $3.57 \pm 326.70$ & $3.52 \pm 216.60$ & $2.82 \pm 155.73$ & $2.02 \pm 101.66$
\end{tabular}
D
C
D C
D
B
( kg/ 75mg)T5

\begin{tabular}{|c|c|c|c|c|c|}
$1.96 \pm 1116.70$ & $0.80 \pm 396.70$ & $1.99 \pm 256.70$ & $2.01 \pm 210.00$ & $2.12 \pm 146.70$ & $1.52 \pm 106.60$ \\
\hline
\end{tabular}

الحروف المختلفة عموديا تثير الى وجود فروق معنوية بين متوسطات المعاملات عند مستوى احتمال (p0.01)

جدول, 4: تأثير اضافة نسب مختلفة من مسحوق كبريتات الزنك الى العليقة في معدل استهلاك العلف (غم/يوم) لفروج اللحم عند الاعمار المختلفة

\begin{tabular}{|c|c|c|c|c|c|c|}
\hline 42 & 35 & 28 & 21 & 14 & 7 & المباليوم / المعامة \\
\hline $\begin{array}{c}C \\
13.82 \pm 3296.88\end{array}$ & $\begin{array}{c}C \\
10.19 \pm 1183.95\end{array}$ & $\begin{array}{c}\text { B } \\
25.12 \pm 876.09\end{array}$ & $\begin{array}{c}\text { B } \\
8.52 \pm 576.26\end{array}$ & $\begin{array}{c}\text { CD } \\
5.54 \pm 374.92\end{array}$ & $\begin{array}{c}\text { B } \\
8.13 \pm 285.64\end{array}$ & $\begin{array}{c}\text { T1 } \\
\text { (control) }\end{array}$ \\
\hline $\begin{array}{c}\text { D } \\
20.43 \pm 3098.86\end{array}$ & $\begin{array}{c}\text { D } \\
8.74 \pm 1082.69\end{array}$ & $\begin{array}{c}\mathrm{C} \\
9.92 \pm 827.51\end{array}$ & $\begin{array}{c}\text { B } \\
0.32 \pm 566.37\end{array}$ & $\begin{array}{c}\text { D } \\
5.95 \pm 365.05\end{array}$ & $\begin{array}{c}C \\
4.51 \pm 257.18\end{array}$ & $\begin{array}{c}30 \text { )T2 } \\
(\mathrm{kg} / \mathrm{mg}\end{array}$ \\
\hline $\begin{array}{c}\text { CD } \\
5.17 \pm 3207.0033\end{array}$ & $\begin{array}{c}\text { D } \\
21.37 \pm 1080.84\end{array}$ & $\begin{array}{c}\text { B } \\
23.35 \pm 871.87\end{array}$ & $\begin{array}{c}B \\
6.85 \pm 580.74\end{array}$ & $\begin{array}{c}\text { B } \\
3.93 \pm 400.61\end{array}$ & $\begin{array}{c}\text { BC } \\
5.83 \pm 272.93\end{array}$ & $\underset{(\mathrm{kg} /}{\mathrm{mg45}) \mathrm{T3}}$ \\
\hline $\begin{array}{c}\text { B } \\
7.91 \pm 3681.39\end{array}$ & $\begin{array}{c}\text { B } \\
9.61 \pm 1360.8\end{array}$ & $\begin{array}{c}\mathrm{A} \\
8.95 \pm 1028.44\end{array}$ & $\begin{array}{c}\text { B } \\
5.51 \pm 630.67\end{array}$ & $\begin{array}{c}\text { BC } \\
12.01 \pm 390.71\end{array}$ & $\begin{array}{c}\text { BC } \\
3.38 \pm 270.76\end{array}$ & $\begin{array}{c}\text { mg60)T4 } \\
(\mathrm{kg} /\end{array}$ \\
\hline $\begin{array}{c}\mathrm{A} \\
7.50 \pm 4057.38\end{array}$ & $\begin{array}{c}\text { A } \\
\mathbf{3 0 . 4 4} \pm 1558.85\end{array}$ & $\begin{array}{c}\text { B } \\
7.20 \pm 934.82\end{array}$ & $\begin{array}{c}\text { A } \\
15.37 \pm 751.94\end{array}$ & $\begin{array}{c}\mathrm{A} \\
5.23 \pm 493.24\end{array}$ & $\begin{array}{c}\text { A } \\
12.86 \pm 318.52\end{array}$ & $\begin{array}{c}75 \mathrm{mg}) \mathrm{T} 5 \\
(\mathrm{~kg} /\end{array}$ \\
\hline
\end{tabular}
الحروف المختلفة عموديا تثبير الى وجود فروق معنوية بين متوسطات المعاملات عند مستوى احتمال (p) (p.01)

جدول 5: تأثير اضافة نسب مختلفة من مسحوق كبريتات الزنك في العليقة على معامل التحويل الغذائي (غم) علف مستهلك لكل غم زياده وزنية لفروج

\begin{tabular}{|c|c|c|c|c|c|c|}
\hline 42 & 35 & 28 & 21 & 14 & 7 & العمر / باليوم \\
\hline $\begin{array}{c}\mathrm{BC} \\
1.12 \pm 2.56\end{array}$ & $\begin{array}{c}B \\
2.35 \pm 2.71\end{array}$ & $\begin{array}{c}\mathrm{C} \\
8.97 \pm 2.61\end{array}$ & $\begin{array}{c}\mathrm{C} \\
3.37 \pm 2.41\end{array}$ & $\begin{array}{l}\text { C } \\
3.67 \pm 2.24\end{array}$ & $\begin{array}{c}\text { B } \\
9.10 \pm 2.58\end{array}$ & (control)T1 \\
\hline $\begin{array}{c}C \\
1.64 \pm 2.22\end{array}$ & $\begin{array}{c}C \\
2.63 \pm 2.24\end{array}$ & $\begin{array}{c}\mathrm{D} \\
2.13 \pm 2.30\end{array}$ & $\begin{array}{c}D \\
2.23 \pm 2.19\end{array}$ & $\begin{array}{c}D \\
5.96 \pm 2.04\end{array}$ & $\begin{array}{c}\mathrm{C} \\
7.58 \pm 2.24\end{array}$ & (kg/ $30 \mathrm{mg}) \mathrm{T} 2$ \\
\hline $\begin{array}{c}C \\
4.32 \pm 2.43\end{array}$ & $\begin{array}{c}B \\
6.10 \pm 2.54\end{array}$ & $\begin{array}{c}C \\
7.05 \pm 2.51\end{array}$ & $\begin{array}{c}\text { D } \\
3.53 \pm 2.28\end{array}$ & $\begin{array}{c}C \\
6.43 \pm 2.22\end{array}$ & $\begin{array}{c}\text { BC } \\
1.29 \pm 2.41\end{array}$ & ( kg/ mg45)T3 \\
\hline $\begin{array}{c}\text { B } \\
\mathbf{0 . 2 5} \pm \mathbf{2 . 8 7}\end{array}$ & $\begin{array}{c}\text { B } \\
0.49 \pm 2.91\end{array}$ & $\begin{array}{c}\text { B } \\
0.28 \pm 3.15\end{array}$ & $\begin{array}{c}\text { B } \\
0.21 \pm 2.91\end{array}$ & $\begin{array}{c}\text { B } \\
4.19 \pm 2.50\end{array}$ & $\begin{array}{c}\text { B } \\
8.30 \pm 2.66\end{array}$ & (kg/mg60)T4 \\
\hline$\underset{6.32 \pm 3.63}{A}$ & $\stackrel{\mathrm{A}}{8.47 \pm 3.92}$ & $\begin{array}{c}A \\
0.26 \pm 3.63\end{array}$ & $\begin{array}{c}\mathrm{A} \\
5.1 \pm 3.58\end{array}$ & $\underset{6.95 \pm 3.36}{A}$ & $\begin{array}{c}\mathrm{A} \\
\mathbf{0 . 1 4} \pm \mathbf{2 . 9 9}\end{array}$ & $(\mathrm{kg} / 75 \mathrm{mg}) \mathrm{T} 5$ \\
\hline
\end{tabular}

الحروف المختلفة عموديا تثير الى وجود فروق معنوية بين متوسطات المعاملات عند مستوى احتمال (p >

جدول 6, تأثير اضافة نسب مختلفة من مسحوق الزنك في عليقة فروج اللحم في نسبة التصافي عند عمر 7 اسابيع*

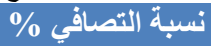

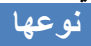

السيطرة المعاملة

B1.62 \pm 72.15

مسحوق الزنتك 30 ملفم/ كغم علف

T1

A1.60 \pm 76.10

مسحوق الزنك 45 ملغم/ كفم علف كف علف

T2

B1.05 \pm 73.69

مسحوق الزنك560 ملفم /كغم علف

T3

B1.35 \pm 72.10

مسحوق الزنتك 75 ملغم/ كفم علف

T5

الحروف المختلفة عموديا تثير الى وجود فروق معنوية بين متوسطات المعاملات عند مستوى احتمال (p0.01) 
15. Bounet, S. P.; Geraet, A.; Lessire, M.; Carre, B. and Guillaumin, S.(1997). Effect of high ambient temperature on feed digestibility in broilers. Poult. Sci., 76:857-863.

16. Hai, L.; Roug, D. and Zhang, Z.Y.(2000). The effect of thermal environment on the digestion of broilers. J. Anim. Physiol. Anim. Nutr., (Berl.) 83:57-64.

17. Sahin, K. and Kucuk, O. (2003). Heat stress and dietary vitamin supplementation of poultry diets. Nutr. Abstr. Rev. Ser. B Livest. Feeds Feed., 73:41-50.

18. Onderci, M.; Sahin, N.; Sahin, K. and Kilic. N.(2003). The antioxidant properties of chromium and zinc: In vivo effects on digestibility, lipid peroxidation, antioxidant vitamins and some minerals under a low ambient temperature. Biol. Trace Elem. Res., 92:139-150.

19. Deaton, J.W.; Braton, S.L.; Simmous, J.D. and Lott, B.D. (1996). The effect of brooding temperature of broiler performance. Poult. Sci., 75:1217-1220.

20. Salgueiro, M.J.; Zubillaga, M.; Lysionek, A.; Sarabia, M. I.; Caro, R.; De Paoli, T.; Hager, A.;Weill, R. and Boccio, J.(2000). Zinc as essential micronutrient: A review. Nutr. Res., 20:737-755.

21. Oteiza, P. L.; Olin, K. L.; Fraga, C.G. and Keen C. L. (1996). Oxidant defens system in testes from $\mathrm{Zn}$ deficient rats. Proc. Soc. Exp. Biol. Med., 213:85-91.

22. Prasad, A.S. (1993). Biochemistry of zinc. Plenum Press, New York, NY.

23. Powell, S. R. (2000). The antioxidant properties of zinc. J. Nutr., 130:1447S-1454S.

24. Prasad, A. S. (1997). The role of zinc in brain and nerve functions. In Metals and Oxidative Damage in Neurological Disorders. A. Connor, ed. Plenum Press. New York, NY. PP: 95-111.

25. Ensmiuger, M.E.; Oldfield, J.E. and Heinemann, W.W. (1990). Feeds and Nutrition. The Ensming Puplishing Company, Clovis, CA. PP: 8-120.

26. Kucuk, O. (2008). Zinc in a combination with magnesium helps reducing negative effects of heat stress in quails. Biol. Trace Elem. Res., 123:144-153.

27. Halliwell, B.E. and Gutteridge, J. M. (1989). Lipid peroxidation: a radical chain reaction. in

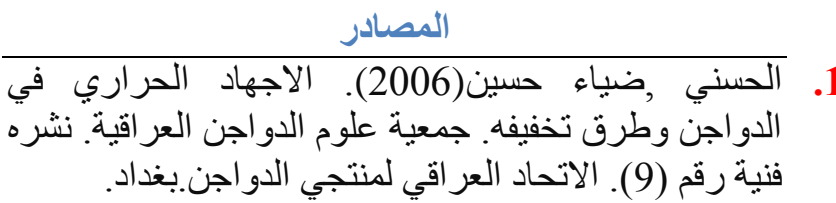

2. Dale, N.M. and Fuller, H.L. (1979). Effects of diet composition on feed intake and growth of chicks under heat stress. Poult. Sci., 58:15291534.

3. Waldroup, P.W.; Mitchell, R.J.; Payne, J. R.; Johnson, Z.B. and Hazen, K.R. (1976). Performance of chicks fed diets formulated to minimize excess levels of essential amino acids. Poult. Sci., 55:243 253.

4. Moreng, R.E.(1980).Temperature and vitamin requirements of the domestic fowl. Poult. Sci. 59:782-785.

5. Nollet, L.; Huyghebaert, G. and Spring, P. (2008). Effect of different levels of dietary organic (bioplex) trace minerals on live performance of broiler chickens by growth phases. J. Appl. Poult. Res., 17:109-115.

6. Bartlett, J.R. and Smith, M.O. (2003). Effects of different levels of zinc on the performance and immunocompetence of broilers under heat stress. Poult. Sci., 82:1580-1588.

7. Prasad, A.S. and Kucuk O.(2002). Zinc in cancer prevention. Cancer Metastasis Rev., 21:291-295.

8. MacDonald, R.S. (2000). The role of zinc in growth and cell proliferation. J. Nutr. 130(5S Suppl.):1500-1508.

9. Roberson, K.D. and Edwards, H. M. (1994). Effects of 1, 25 di-hydroxycholecalciferol and phytase on zinc utilization in broiler chicks. Poult. Sci., 73:1312-1316.

10. Sahin, K., and Kucuk O.(2003). Zinc supplementation alleviates heat stress in laying Japanese quail. J. Nutr. 33:2808-2811.

$$
\begin{aligned}
& \text { 11. الزبيدي، صعيب سعيد علوان ـ 1986. ادارة الدواجن . }
\end{aligned}
$$

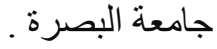

$$
\begin{aligned}
& \text { 12. الفياض، حمدي عبد العزيز و سعد عبد الحسين ناجي . } \\
& \text { 1989. تكنولوجيا منتجات الدواجن ـ الطبعة الاولى ـ مديرية }
\end{aligned}
$$

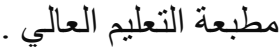

$$
\begin{aligned}
& \text { 13. المحمد , نعيم ثاني , خاشع محمود , يونس , مؤيد احمد , }
\end{aligned}
$$

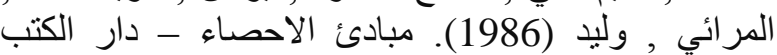

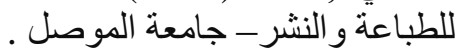

14. NRC.(1994). Nutrient Requirements of poult. $9^{\text {th }}$ rev. ed. National Academy Press, Washington. DC. 
aproducao de frangos decorate em altas temperaturas. In: Encontro Internacional dos Negocio, Cuiaba: enipec; 1 CD-Rom.

30. Jacob, R.A.(1995). The integrated antioxidant system. Nutrition Res., 15(4):755-766.
Free Radicals in Biology and Medicine. $2^{\text {nd }}$ ed. Oxford University Press. New York, NY., PP:188-218

28. Feenster, R. (1985). High temperatures decrease vitamin utilization. Misset. Poult., 38:38-41.

29. Ribeiro, A.M. and Lagana, C. (2002). Estrategies nutricionais para otimizar

\section{Effects of Ration Zinc Supplementation on Some Productive Characters on Heat Stressed Broiler}

\section{Mohammad Katih Manhoob; Raad Hatem; Hayder Razooqi Kadhem Shkeer; Mohammad Jabar Ahmed and Aqeel Abdallh Razooqi}

The Agricultural Research Service, Ministry of Science and Technology, Iraq

\section{Summary}

The objective of the study was to investigate the effect of using different dietary levels of zink on the performance of broiler reared during summer months, under heat stress by using 120 Ross broiler chicks one day of age were used. The birds were randomly distributed to five treatment with three replicates per treatment ( 8 chicks /replicated). Zinc was supplemented into ration from the second week until the end of experiment (42 days) at levels 0(T1), 30(T2), 45(T3), 60(T4) and 75 (T5) $\mathrm{mg} / 1 \mathrm{~kg}$ of ration. The result of statistical analysis showed that birds in T2 and T3 had significantly $(\mathrm{P}<0.01)$ high body weight gained, food conversion ratio when compared with all treatments. In conclusion of this study indicated that ration zinc supplementation increased the resistance of broiler into heat stress.

Keywords: Broiler, Zinc, Heat stress. 